\title{
Cardiac Safety of Esketamine Nasal Spray in Treatment-Resistant Depression: Results from the Clinical Development Program
}

\author{
Teodora Doherty ${ }^{1}$ Ewa Wajs $^{2} \cdot$ Rama Melkote $^{3} \cdot$ Janice Miller $^{1} \cdot$ Jaskaran B. Singh $^{4,6} \cdot$ Michael A. Weber $^{5}$
}

Published online: 29 January 2020

(c) The Author(s) 2020

\begin{abstract}
Background An intranasal formulation of esketamine, combined with an oral antidepressant, is approved in the USA and the European Union for adults with treatment-resistant depression (TRD). Transient cardiovascular stimulatory effects have been reported with ketamine.

Methods Cardiovascular effects of esketamine nasal spray, combined with an oral antidepressant, were evaluated in 1708 esketamine-treated adults with TRD in six trials (five double-blind, placebo-controlled (486 placebo-treated patients); one open-label) of 4-52 weeks' duration. Patients with established cardiovascular disease, including uncontrolled hypertension $(>140 />90 \mathrm{mmHg}$ ), history of hypertensive crisis, or clinically significant electrocardiogram (ECG) abnormalities, were excluded from enrollment. Effects on cardiac repolarization were assessed in a phase I randomized, positive- and activecontrolled thorough corrected QT (QTc) interval study. For adverse events, odds ratio (OR) [95\% confidence interval] for esketamine/antidepressant versus antidepressant/placebo was calculated.

Results Adverse events related to increased BP were reported in 12.8\% of all esketamine-treated patients (in double-blind trials: esketamine/antidepressant $11.6 \%$ vs. antidepressant/placebo 3.9\%; OR 3.2 [1.9-5.8]). Among the patients without a history of hypertension, new antihypertensive medication was initiated by $2.1 \%$ (6/280) of patients in the esketamine/antidepressant group versus $1.2 \%$ (2/171) of patients in the antidepressant/placebo group, in the double-blinded studies. Adverse events related to abnormal heart rate were reported in 3.0\% of all esketamine-treated patients (in double-blind trials: $1.6 \%$ vs. $0.8 \%$; OR 1.9 [0.5-8.6]). Overall, three cardiovascular adverse events related to BP increase were reported as serious and severe, and there was one fatal event considered unrelated (acute cardiac failure). BP increases reached the maximum postdose value within $\sim 40$ min of esketamine dosing and returned to the predose range by $\sim 1.5 \mathrm{~h}$ postdose. In two studies (4-week duration, age 18-64 years), the largest mean maximum systolic/diastolic postdose BP increases were 13.3/8.7 mmHg for esketamine/antidepressant and 6.1/4.9 mmHg for antidepressant/placebo, and in a short-term elderly study (age $\geq 65$ years) were 16.0/9.5 and 11.1/6.8 $\mathrm{mmHg}$, respectively. Across studies/study phases, $<2 \%$ of patients discontinued esketamine due to adverse events of increased BP and tachycardia. No clinically relevant effect on ECG parameters was observed. Therapeutic and supratherapeutic doses of esketamine did not prolong the QTcF (QT corrected by Fridericia's equation) interval (baseline-corrected values of -2.02 to $2.16 \mathrm{~ms}$, and -3.51 to $4.89 \mathrm{~ms}$, respectively). Conclusions BP elevations following esketamine dosing are generally transient, asymptomatic, and not associated with serious cardiovascular safety sequalae. Further evaluation of long-term cardiovascular outcomes is warranted.
\end{abstract}

\section{Introduction}

Ketamine, an antagonist of the glutamatergic $N$-methyl$D$-aspartate (NMDA) receptor (an ionotropic glutamate receptor), was shown to exhibit rapid onset and robust, short-term antidepressant effect in patients with treatmentresistant depression (TRD) [1-3]. Esketamine, the $S$-enantiomer of ketamine racemate, was recently approved by the

Teodora Doherty

tdoherty@its.jnj.com

Extended author information available on the last page of the article
United States (US) Food and Drug Administration (FDA) and by the European Medicines Agency (EMA) as an intranasal formulation for adults with TRD based on efficacy and safety findings from short-term phase II/III studies [4-9] and longer-term phase III studies [7, 8].

Transient cardiovascular stimulatory effects have been reported with ketamine, beginning shortly after dosing for general anesthesia [at doses of $0.5-2 \mathrm{mg} / \mathrm{kg}$ intravenously (IV)], including increase in arterial blood pressure (BP), heart rate, and cardiac output [10]. Although ketamine has direct cardio-depressant and negative inotropic effects, the indirect sympathomimetic effect dominates in physiological 


\section{Key Points}

In a large cohort $(N=1708)$ of patients with treatmentresistant depression who had no clinically important cardiovascular disease or had hypertension controlled by antihypertensive medications, adverse events of esketamine nasal spray were transient and manageable in a clinical setting under the observation of a healthcare professional.

In general, blood pressure elevations following dosing of esketamine nasal spray were short-lived, were not associated with symptoms, rarely required treatment with antihypertensive medication, and did not result in serious cardiovascular safety sequalae.

Esketamine nasal spray has no clinically relevant treatment-emergent effect on the duration of mean electrocardiogram intervals.

conditions [11]. This is triggered by a systemic release of catecholamines, inhibiting the vagal nerve and inhibiting norepinephrine and epinephrine reuptake at peripheral nerves and within the myocardium [11]. While the exact mechanism is unknown, ketamine is considered to have sympathomimetic effects by influencing calcium influx [12].

In a recently published study, IV infusion of ketamine $(0.5 \mathrm{mg} / \mathrm{kg}$ over $40 \mathrm{~min})$ in patients with major depressive disorder was associated with mean systolic BP (SBP) and diastolic BP (DBP) increase by $\sim 3 \mathrm{mmHg}$ at peak, 30 min into the infusions, and returned toward baseline levels shortly thereafter [13]. Wan et al. [14] reported higher transient BP increases (peak increase of 19.6/13.4 mmHg based on 205 ketamine infusions) [14]. These cardiovascular effects may complicate treatment of patients with TRD who, in real-world settings, often have hypertension (67\%) and cardiovascular disease (56\%) [15].

It is important to characterize the cardiovascular effects of esketamine, given that patients with major depression/TRD are at increased risk of cardiac morbidity/mortality [16, 17]. We report herein the cardiovascular effects of esketamine nasal spray, adjunctive to an oral antidepressant, in a large cohort of patients with TRD who were treated in the esketamine clinical development program.

\section{Materials and Methods}

\subsection{Study Design}

The cardiovascular safety analysis population came from five double-blind, placebo-controlled, phase II/III studies and one open-label phase III study of esketamine nasal spray in adults with TRD (Table 1 [4-9]).

\subsection{Study Population}

All studies enrolled patients with moderate-to-severe depression, without psychotic features, who met the study definition of TRD (i.e., non-response to two separate and adequate trials of an antidepressant in the current episode of depression, of which one was observed prospectively). Two studies enrolled elderly patients (age $\geq 65$ years), and five studies enrolled patients between 18 and 64 years of age. Of note with regard to cardiac effects, patients with cardiovascular disease (cerebrovascular disease with a history of stroke or transient ischemic attack; aneurysmal vascular disease; coronary artery disease with myocardial infarction, unstable angina, or revascularization procedure within 12 months before the start of the trial; hemodynamically significant valvular heart disease such as mitral regurgitation, aortic stenosis, or aortic regurgitation; New York Heart Association Class III-IV heart failure), uncontrolled hypertension ( $>140 />90 \mathrm{mmHg}$ ), history of hypertensive crisis, or clinically significant electrocardiogram (ECG) abnormalities were excluded from enrollment.

\subsection{Study Drug}

Eligible patients received esketamine nasal spray $(28 \mathrm{mg}$ (only for patients 65 years and older) to $84 \mathrm{mg}$ twice weekly, once weekly, or every other week; Table 1) in combination with an oral antidepressant for 4-52 weeks. In the phase III studies [5-9], patients received a new oral antidepressant [one of the following options: a selective serotonin reuptake inhibitor (SSRI: escitalopram or sertraline) or a serotonin and norepinephrine reuptake inhibitor (SNRI: duloxetine or venlafaxine extended release)], which was initiated at the start of the induction phase, whereas in the adjunctive phase II study [4], no new antidepressant was initiated and patients were permitted to continue their ongoing oral antidepressant treatment. In the placebo-controlled studies, patients randomized to placebo received intranasal study drug [i.e., water for injection with bittering agent (to imitate the bitter taste of esketamine) and a preservative] that was provided in a disposable nasal spray device with identical appearance and packaging to that of esketamine nasal spray. Patients self-administered the intranasal study drug at the study site under the direct supervision of a site staff member.

\subsection{Cardiac Safety Assessments}

Adverse events (including severity, seriousness, and outcomes), vital signs, and ECG were monitored throughout the studies. Severity of adverse events was graded as mild 


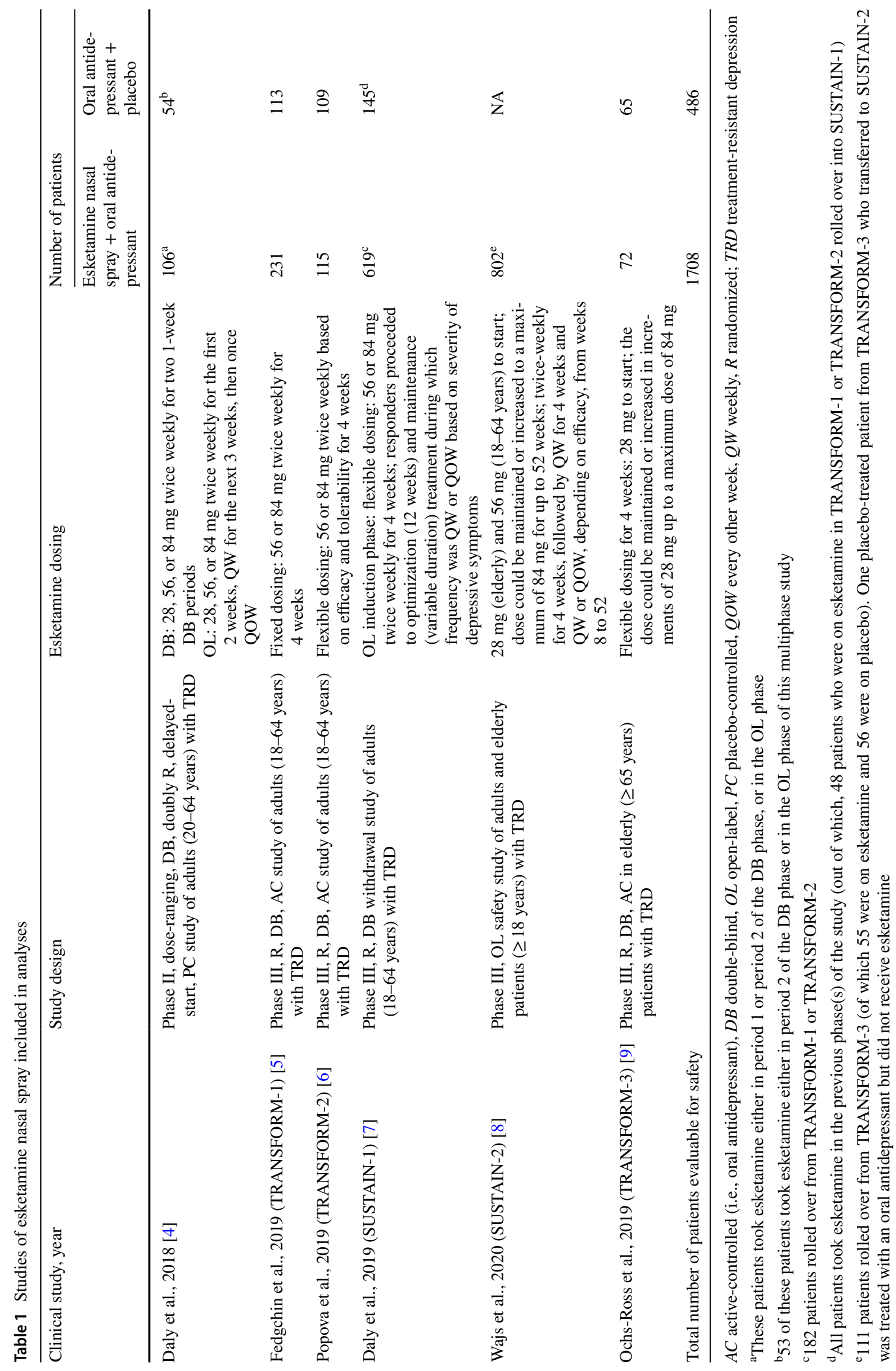


(symptoms were easily tolerated, caused minimal discomfort, and did not interfere with everyday activities); moderate (discomfort caused interference with normal activity); or severe (extreme distress caused significant impairment of functioning or incapacitation, and prevented normal everyday activities).

Vital signs were measured at baseline and all dosing visits (phase II: predose, and at $40 \mathrm{~min}$ and $2 \mathrm{~h}$ postdose; phase III: predose, and at $40 \mathrm{~min}, 1 \mathrm{~h}$, and $1.5 \mathrm{~h}$ postdose). $\mathrm{BP}$ and pulse/heart rate measurements were assessed after the patient had rested for at least $5 \mathrm{~min}$ and were measured supine, either with a completely automated device or using manual techniques.

In the phase III studies, as a risk mitigation measure, guidance on BP monitoring on intranasal treatment days was provided to investigators [i.e., no dosing if predose SBP $>140 \mathrm{mmHg}$ ( $>150$ for age $>65$ years) or DBP $>90 \mathrm{mmHg}$; dose interruption if postdose $\mathrm{SBP} \geq 200 \mathrm{mmHg}$ ( $\geq 190$ for age $>65$ years) or DBP $\geq 110 \mathrm{mmHg}$ ( $\geq 100$ for age $>65$ years)]; patients with elevated BPs were referred to a specialist or general practitioner for evaluation and potential treatment [18].

Single, 12-lead ECGs were performed at pre-specified timepoints after patients had rested in a supine position for at least $5 \mathrm{~min}$. All ECG tracings were sent to a central ECG laboratory and were read by a cardiologist.

The effects of esketamine on cardiac repolarization were assessed in a phase I randomized, positive- and activecontrolled (oral moxifloxacin $400 \mathrm{mg}$ ) thorough corrected QT (QTc) interval study. Healthy subjects were randomly assigned in a double-blind, crossover manner to single doses of placebo, oral moxifloxacin, or esketamine $84 \mathrm{mg}$ nasal spray in the first three periods, and to esketamine $0.8 \mathrm{mg} / \mathrm{kg}$ as a 40-min IV infusion (supratherapeutic dose) in the fourth period. Placebo was given in combination with each of the active treatments.

\subsection{Statistical Methods}

Safety data were analyzed in an All Randomized, Blinded Trials TRD Population [4-7, 9] and in an All Clinical Trials TRD Population [4-9], both including patients who received at least one dose of intranasal study drug.

Increased BP-related adverse events included the following Medical Dictionary for Regulatory Activities $\left(\right.$ MedDRA $^{\circledR}$ ) (version 20.0) preferred terms: accelerated hypertension; blood pressure abnormal; blood pressure ambulatory abnormal; blood pressure ambulatory increased; blood pressure diastolic abnormal; blood pressure diastolic increased; blood pressure inadequately controlled; blood pressure increased; blood pressure orthostatic abnormal; blood pressure orthostatic increased; blood pressure systolic abnormal; blood pressure systolic increased; diastolic hypertension; hypertension; hypertensive cerebrovascular disease; hypertensive crisis; hypertensive emergency; hypertensive encephalopathy; hypertensive end-organ damage; hypertensive heart disease; labile hypertension; orthostatic hypertension; prehypertension; supine hypertension; and systolic hypertension.

Adverse events related to abnormal heart rate included the following MedDRA ${ }^{\circledR}$ (version 20.0) preferred terms: atrial fibrillation; atrial tachycardia; cardiac fibrillation; extrasystoles; heart rate abnormal; heart rate increased; palpitations; sinus tachycardia; sudden cardiac death; sudden death; supraventricular tachycardia; tachyarrhythmia; tachycardia; tachycardia paroxysmal; and ventricular tachycardia.

Cardiovascular adverse events of clinical interest included, but were not limited to, the following MedDRA ${ }^{\circledR}$ (version 20.0) preferred terms: angina pectoris; cardiac failure acute; chest discomfort; chest pain; and hypertensive heart disease.

\subsubsection{Analyses}

For adverse events, an odds ratio (OR) and a two-sided exact 95\% confidence interval (CI) for esketamine/antidepressant versus oral antidepressant/placebo were calculated for the All Randomized, Blinded Trials TRD Population. Results of all other analyses were summarized descriptively.

\subsubsection{Sample Size Determination}

The sample size planned for each study included in the analyses was calculated based on assumptions on efficacy.

\section{Results}

A total of 1708 esketamine nasal spray-treated patients received at least one dose of esketamine in the six completed phase II and III studies, and were included in the All Clinical Trials TRD Population. Of these, 194 (11.4\%) were $\geq 65$ years. The combined cumulative exposure to esketamine nasal spray in the six phase II/III TRD studies was 611 patient-years (601 patient-years in the phase III studies), and the combined cumulative exposure to placebo nasal spray was 108 patient-years (107 patient-years in the phase III studies). Of the 1601 patients exposed to esketamine nasal spray across the five completed phase III studies, 479 were exposed to esketamine for at least 6 months and 178 were exposed for at least 12 months.

Baseline demographic and disease characteristics for the All Clinical Trials TRD Population are presented in Table 2.

Increased BP reported as an adverse event occurred in $12.8 \%$ of all esketamine-treated patients (Table 3 ). In the double-blind trials, the rate of increased BP-related adverse 
Table 2 Demographic and baseline characteristics of patients in phase II/III studies of esketamine nasal spray—all clinical trials TRD population $^{\mathrm{a}}$

\begin{tabular}{|c|c|}
\hline Characteristic & $\begin{array}{l}\text { Esketamine }+ \text { oral } \\
\text { antidepressant } \\
(N=1708)\end{array}$ \\
\hline \multicolumn{2}{|l|}{ Age (years) } \\
\hline Mean (SD) & $49.1(12.91)$ \\
\hline Range & $18-86$ \\
\hline \multicolumn{2}{|l|}{$\operatorname{Sex}[n(\%)]$} \\
\hline Male & $634(37.1)$ \\
\hline Female & $1074(62.9)$ \\
\hline \multicolumn{2}{|l|}{$\operatorname{Race}[n(\%)]$} \\
\hline Asian & $126(7.4)$ \\
\hline Black or African American & $72(4.2)$ \\
\hline White & $1445(84.6)$ \\
\hline Multiple & $13(0.8)$ \\
\hline Other & $30(1.8)$ \\
\hline Not reported/unknown & $22(1.3)$ \\
\hline \multicolumn{2}{|c|}{ Baseline body mass index $\left(\mathrm{kg} / \mathrm{m}^{2}\right)$} \\
\hline Mean (SD) & $28.1(6.00)$ \\
\hline Range & $16-67$ \\
\hline \multicolumn{2}{|c|}{ Age when diagnosed with MDD $^{\mathrm{b}}$ (years) } \\
\hline Mean (SD) & $33.9(12.97)$ \\
\hline Range & $5-75$ \\
\hline \multicolumn{2}{|c|}{ Duration of current episode $^{\mathrm{b}}$ (weeks) } \\
\hline Mean (SD) & $148.7(238.4)$ \\
\hline Range & $4-2288$ \\
\hline \multicolumn{2}{|l|}{ Hypertension status $[n(\%)]$} \\
\hline Yes & $403(23.6)$ \\
\hline No & $1305(76.4)$ \\
\hline
\end{tabular}

$M D D$ major depressive disorder, $S D$ standard deviation

${ }^{a}$ Includes safety evaluable patients from studies cited in Daly et al. [4, 7], Fedgchin et al. [5], Popova et al. [6], Wajs et al. [8], and OchsRoss et al. [9]

${ }^{\mathrm{b}} N=1705$

events was $\sim 3$-fold higher in the esketamine/antidepressant group versus the antidepressant/placebo group (11.6\% vs. 3.9\%; OR 3.2 [95\% CI 1.9-5.8]); most of the increased BPrelated adverse events were considered as mild or moderate (98\%) and resolved (94\%). BP elevation following initial administrations of study drug did not predict BP elevation following later administrations (data not presented).

Adverse events related to abnormal heart rate (e.g., palpitations, tachycardia) were reported in $3.0 \%$ of all esketamine-treated patients (Table 4). In the double-blind trials, the rate of adverse events related to abnormal heart rate was $\sim 2$-fold higher in the esketamine/antidepressant versus the antidepressant/placebo group (1.6\% vs. 0.8\%; OR 1.9 [95\% CI 0.5-8.6]); most of the events were mild or moderate $(96 \%)$ and resolved (88\%).
Cardiovascular adverse events of clinical interest were uncommon ( $1.3 \%$ of all esketamine-treated patients), and were reported at a rate that was similar between the esketamine/antidepressant and antidepressant/placebo groups in double-blind studies (Table 5).

In general, increased BP-related adverse events, abnormal heart rate-related adverse events, and cardiovascular adverse events of clinical interest experienced by esketamine-treated patients had an onset shortly after dosing and resolved by $1.5 \mathrm{~h}$ postdose while patients were in the clinic.

In the all-clinical trials TRD population, three serious and severe cardiovascular adverse events related to BP increase were reported for two patients. One event was reported for a 74-year-old male patient whose BP increased from 130/90 to $170 / 110 \mathrm{mmHg}$ on day $1,5 \mathrm{~min}$ after administration of a $28 \mathrm{mg}$ dose of esketamine, and resolved on the same day. Hypertensive crisis and sinus tachycardia, which began 3 days after the last dose of esketamine and resolved 2 days later, were reported for the second patient, who had a history of hypertension and panic attacks. Cardiac enzymes, chest radiograph, computed tomography angiogram, and echocardiogram for the patient were all normal at the time of the event. The differential diagnosis included a hypertensive episode due to non-compliance with concomitant lisinopril and severe panic attack.

In addition to the above-described cases, a serious and severe unrelated event of lacunar stroke was reported for a 64-year-old female patient with a history of hypercholesterolemia on day 1, $6 \mathrm{~h}$ after esketamine nasal spray administration. Magnetic resonance imaging confirmed the ischemic character of the lesion. Given the absence of findings suggestive of an intracranial hemorrhage, the plausible mechanism for this ischemic stroke was considered to be microatheroma. A non-serious adverse event of increased BP was also reported for this patient on day 1 . Her BP increased from $116 / 57 \mathrm{mmHg}$ prior to esketamine dosing to $162 / 74 \mathrm{mmHg}$ at $40 \mathrm{~min}$ postdose, then decreased to $122 / 58 \mathrm{mmHg}$ at $1 \mathrm{~h}$ postdose.

One fatal unrelated event of acute cardiac failure (accompanied by respiratory failure) was reported 5 days after the last dose of esketamine for a 60-year-old male patient with a history of hypertension and obesity. Pulse oximetry, respiratory rate, and BP values for this patient remained normal during his participation in the study; transient nausea and headache, during the first week of the study, were the only adverse events reported for this patient.

In addition, three events were reported as severe and not serious (palpitations for one patient and BP increase for two patients).

In general, BP increases reached a maximum within 40 min of esketamine dosing (consistent with peak plasma concentrations [18]) and typically returned to the predose range by $1.5 \mathrm{~h}$ postdose. Mean $\mathrm{BP}$ over time and mean 
Table 3 Summary of increased blood pressure-related adverse events in esketamine nasal spray phase II/III clinical development studies

\begin{tabular}{|c|c|c|c|}
\hline \multirow[t]{2}{*}{ Adverse events } & \multicolumn{2}{|c|}{ All randomized, blinded trials TRD population ${ }^{a}$} & \multirow{2}{*}{$\begin{array}{l}\text { All clinical } \\
\text { trials TRD } \\
\text { population }^{\mathrm{b}} \\
\text { Esketamine + } \\
\text { oral anti- } \\
\text { depressant } \\
(N=1708)\end{array}$} \\
\hline & $\begin{array}{l}\text { Esketamine }+ \text { oral antidepressant } \\
(N=571)\end{array}$ & $\begin{array}{l}\text { Oral antidepressant }+ \text { placebo } \\
(N=486)\end{array}$ & \\
\hline \multicolumn{4}{|c|}{ Increased blood pressure-related adverse events ${ }^{\mathrm{c}}$} \\
\hline$n(\%)$ & $66(11.6)$ & $19(3.9)$ & $219(12.8)$ \\
\hline Odds ratio & 3.2 & & \\
\hline $95 \% \mathrm{CI}^{\mathrm{d}}$ & $1.9-5.8$ & & \\
\hline \multicolumn{4}{|c|}{ Increased blood pressure-related events (preferred terms) $[n(\%)]$} \\
\hline Blood pressure diastolic increased & $6(1.1)$ & 0 & $30(1.8)$ \\
\hline Blood pressure increased & $51^{\mathrm{e}}(8.9)$ & $15(3.1)$ & $168^{\mathrm{e}}(9.8)$ \\
\hline Blood pressure systolic increased & $4(0.7)$ & $1(0.2)$ & $19(1.1)$ \\
\hline Hypertension & $11(1.9)$ & $3(0.6)$ & $43(2.5)$ \\
\hline Hypertensive crisis & 0 & 0 & $1^{\mathrm{f}}(0.1)$ \\
\hline Hypertensive heart disease & 0 & 0 & $1(0.1)$ \\
\hline \multicolumn{4}{|l|}{ Seriousness $^{\mathrm{g}}[n(\%)]$} \\
\hline Fatal & 0 & 0 & 0 \\
\hline Serious & $1(1.4)$ & 0 & $2(0.8)$ \\
\hline \multicolumn{4}{|l|}{ Outcomes $^{\mathrm{g}}[n(\%)]$} \\
\hline Recovered & $65(90.3)$ & $19(100.0)$ & $238(90.8)$ \\
\hline Recovered with sequelae & $2(2.8)$ & 0 & $3(1.1)$ \\
\hline Recovering & $1(1.4)$ & 0 & $6(2.3)$ \\
\hline Not recovered & $4(5.6)$ & 0 & $15(5.7)$ \\
\hline \multicolumn{4}{|l|}{ Severity $^{\mathrm{g}}[n(\%)]$} \\
\hline Mild & $51(70.8)$ & $15(78.9)$ & $180(68.7)$ \\
\hline Moderate & $20(27.8)$ & $4(21.1)$ & $78(29.8)$ \\
\hline Severe & $1(1.4)$ & 0 & $4(1.5)$ \\
\hline
\end{tabular}

$C I$ confidence interval, $T R D$ treatment-resistant depression

${ }^{a}$ Includes safety evaluable patients from studies cited in Daly et al. [4, 7], Fedgchin et al. [5], Popova et al. [6], and Ochs-Ross et al. [9]

${ }^{b}$ Includes safety evaluable patients from studies cited in references Daly et al. [4, 7], Fedgchin et al. [5], Popova et al. [6], Wajs et al. [8], and Ochs-Ross et al. [9]

${ }^{\mathrm{c}}$ Includes all patients who had $\geq 1$ occurrences of an adverse event that coded to the MedDRA ${ }^{\circledR}$ preferred terms grouped under 'Increased Blood Pressure-related Events'; the patient is counted only once regardless of the number of events or the number of occurrences

${ }^{\mathrm{d}}$ The two-sided exact $95 \%$ CI in odds ratio of esketamine + oral antidepressant to oral antidepressant + placebo for All Randomized, Blinded Trials TRD Population

${ }^{\mathrm{e}}$ One event was severe and serious; 2 events in the All Clinical Trials TRD Population were severe and non-serious

${ }^{\mathrm{f}}$ Event was severe and serious

${ }^{\mathrm{g}}$ The total number of distinct preferred terms (i.e., preferred terms that refer to separate adverse events reported by individual patients) in the 'Increased Blood Pressure-related Events' group by seriousness/outcome and also by severity

maximum and maximum change from baseline in postdose BP in the 4-week double-blind induction phase of TRANSFORM-1 and TRANSFORM-2 are presented in Figs. 1 and 2 , respectively.

Approximately $8-17 \%$ of esketamine-treated patients and $1-3 \%$ of placebo-treated patients experienced an increase of more than $40 \mathrm{mmHg}$ in SBP and/or $25 \mathrm{mmHg}$ in DBP in the first $1.5 \mathrm{~h}$ after administration at least once during the first
4 weeks of treatment. A substantial increase in BP could occur after any dose administered even if smaller BP effects were observed with previous administrations.

The largest mean maximum SBP/DBP increases from predose at any postdose timepoint across all intranasal dosing days were 13.3/8.7 $\mathrm{mmHg}$ for esketamine/antidepressant and 6.1/4.9 $\mathrm{mmHg}$ for antidepressant/placebo in the two 4-week studies of patients aged 18-64 years (Fig. 2) 
Table 4 Summary of adverse events related to abnormal heart rate in esketamine nasal spray phase II/III clinical development studies

\begin{tabular}{|c|c|c|c|}
\hline \multirow[t]{2}{*}{ Adverse events } & \multicolumn{2}{|c|}{ All randomized, blinded trials TRD population ${ }^{\mathrm{a}}$} & \multirow{2}{*}{$\begin{array}{l}\text { All clinical } \\
\text { trials TRD } \\
\text { population }^{\mathrm{b}} \\
\text { Esketamine + } \\
\text { oral anti- } \\
\text { depressant } \\
(N=1708)\end{array}$} \\
\hline & $\begin{array}{l}\text { Esketamine }+ \text { oral antidepressant } \\
(N=571)\end{array}$ & $\begin{array}{l}\text { Oral antidepressant }+ \text { placebo } \\
(N=486)\end{array}$ & \\
\hline \multicolumn{4}{|c|}{ Adverse events related to abnormal heart rate ${ }^{c}$} \\
\hline$n(\%)$ & $9(1.6)$ & $4(0.8)$ & $52(3.0)$ \\
\hline Odds ratio & 1.9 & & \\
\hline $95 \% \mathrm{CI}^{\mathrm{d}}$ & $0.5-8.6$ & & \\
\hline \multicolumn{4}{|c|}{ Adverse events related to abnormal heart rate (preferred terms) $[n(\%)]$} \\
\hline Atrial fibrillation & 0 & 0 & $2(0.1)$ \\
\hline Extrasystoles & $2(0.4)$ & 0 & $3(0.2)$ \\
\hline Heart rate increased & $1(0.2)$ & 0 & $3(0.2)$ \\
\hline Palpitations & $3^{\mathrm{e}}(0.5)$ & $2(0.4)$ & $26^{\mathrm{e}}(1.5)$ \\
\hline Sinus tachycardia & 0 & $1(0.2)$ & $3^{\mathrm{f}}(0.2)$ \\
\hline Tachycardia & $4(0.7)$ & $1(0.2)$ & $20(1.2)$ \\
\hline \multicolumn{4}{|l|}{ Seriousness $^{\mathrm{g}}[n(\%)]$} \\
\hline Fatal & 0 & 0 & 0 \\
\hline Serious & 0 & 0 & $1(1.8)$ \\
\hline \multicolumn{4}{|l|}{ Outcomes $^{\mathrm{g}}[n(\%)]$} \\
\hline Recovered & $8(80.0)$ & $3(75.0)$ & $48(84.2)$ \\
\hline Recovered with sequelae & 0 & 0 & $1(1.8)$ \\
\hline Recovering & 0 & 0 & $1(1.8)$ \\
\hline Not recovered & $2(20.0)$ & $1(25.0)$ & $7(12.3)$ \\
\hline \multicolumn{4}{|l|}{ Severity $^{\mathrm{g}}[n(\%)]$} \\
\hline Mild & $5(50.0)$ & $3(75.0)$ & $43(75.4)$ \\
\hline Moderate & $4(40.0)$ & $1(25.0)$ & $12(21.1)$ \\
\hline Severe & $1(10.0)$ & 0 & $2(3.5)$ \\
\hline
\end{tabular}

$C I$ confidence interval, $T R D$ treatment-resistant depression

${ }^{a}$ Includes safety evaluable patients from studies cited in references Daly et al. [4, 7], Fedgchin et al. [5], Popova et al. [6], and Ochs-Ross et al. [9]

${ }^{b}$ Includes safety evaluable patients from studies cited in Daly et al. [4, 7], Fedgchin et al. [5], Popova et al. [6], Wajs et al. [8], and Ochs-Ross et al. [9]

${ }^{\mathrm{c}}$ Includes all patients who had $\geq 1$ occurrences of an adverse event that coded to the MedDRA ${ }^{\circledR}$ preferred terms grouped under 'Adverse events related to abnormal heart rate'; the patient is counted only once regardless of the number of events or the number of occurrences

${ }^{\mathrm{d}}$ The two-sided exact $95 \% \mathrm{CI}$ in odds ratio of esketamine + oral antidepressant to oral antidepressant + placebo for All Randomized, Blinded Trials TRD Population

${ }^{\mathrm{e}}$ One event was severe and non-serious

${ }^{\mathrm{f}}$ One event was severe and serious

${ }^{\mathrm{g}}$ The total number of distinct preferred terms (i.e., preferred terms that refer to separate adverse events reported by individual patients) in the 'Adverse events related to abnormal heart rate' group by seriousness/outcome and also by severity

and 16.0/9.5 and 11.1/6.8 $\mathrm{mmHg}$, respectively, in the elderly study (TRANSFORM-3).

The percentage of patients (age 18-64 years) with markedly abnormal BP elevation (SBP $\geq 180 \mathrm{mmHg}$ and/or DBP $\geq 110 \mathrm{mmHg}$ ) ranged $2.0-4.9 \%$ in esketamine/antidepressant group versus $0-0.9 \%$ in antidepressant/placebo group across studies/phases and was higher in patients with (5.5-7.6\%) versus without $(4.1-4.3 \%)$ a history of hypertension. The percentages of patients with markedly abnormal BP elevation were higher $(11.1 \%$ in esketamine/antidepressant vs. $6.2 \%$ in antidepressant/placebo) in the elderly, and in the study of elderly patients with (14.6\%) versus without (6.5\%) a history of hypertension.

No discontinuations occurred due to adverse events related to increased BP in either the esketamine/antidepressant group or the antidepressant/placebo group in the 
Table 5 Summary of cardiovascular adverse events of clinical interest in esketamine nasal spray phase II/III clinical development studies

\begin{tabular}{|c|c|c|c|}
\hline \multirow[t]{2}{*}{ Adverse events } & \multicolumn{2}{|c|}{ All randomized, blinded trials TRD population ${ }^{\mathrm{a}}$} & \multirow{2}{*}{$\begin{array}{l}\text { All clinical } \\
\text { trials TRD } \\
\text { population }^{\mathrm{b}} \\
\text { Esketamine }+ \\
\text { oral anti- } \\
\text { depressant } \\
(N=1708)\end{array}$} \\
\hline & $\begin{array}{l}\text { Esketamine }+ \text { oral antidepressant } \\
(N=571)\end{array}$ & $\begin{array}{l}\text { Oral antidepressant }+ \text { placebo } \\
(N=486)\end{array}$ & \\
\hline \multicolumn{4}{|c|}{ Cardiovascular adverse events of clinical interest ${ }^{\mathrm{c}}$} \\
\hline$n(\%)$ & $3(0.5)$ & $2(0.4)$ & $23(1.3)$ \\
\hline Odds ratio & 1.3 & & \\
\hline $95 \% \mathrm{CI}^{\mathrm{d}}$ & $0.1-15.4$ & & \\
\hline \multicolumn{4}{|c|}{ Cardiovascular events of clinical interest (preferred terms) $[n(\%)]$} \\
\hline Angina pectoris & 0 & 0 & $1(0.1)$ \\
\hline Cardiac failure acute & 0 & 0 & $1^{\mathrm{e}}(0.1)$ \\
\hline Chest discomfort & $2^{\mathrm{f}}(0.4)$ & 0 & $15^{\mathrm{f}}(0.9)$ \\
\hline Chest pain & $1(0.2)$ & $2(0.4)$ & $5^{\mathrm{g}}(0.3)$ \\
\hline Hypertensive heart disease & 0 & 0 & $1(0.1)$ \\
\hline \multicolumn{4}{|l|}{ Seriousness $^{\mathrm{h}}[n(\%)]$} \\
\hline Fatal & 0 & 0 & $1(4.3)$ \\
\hline Serious & 0 & 0 & $2(8.7)$ \\
\hline \multicolumn{4}{|l|}{ Outcomes $^{\mathrm{h}}[n(\%)]$} \\
\hline Recovered & $3(100)$ & $2(100)$ & $21(91.3)$ \\
\hline Recovered with sequelae & 0 & 0 & 0 \\
\hline Recovering & 0 & 0 & $1(4.3 \%)$ \\
\hline Not recovered & 0 & 0 & 0 \\
\hline \multicolumn{4}{|l|}{ Severity $^{\mathrm{h}}[n(\%)]$} \\
\hline Mild & 0 & $2(100)$ & $12(52.2)$ \\
\hline Moderate & $2(66.7)$ & 0 & $5(21.7)$ \\
\hline Severe & $1(33.3)$ & 0 & $6(26.1)$ \\
\hline
\end{tabular}

$C I$ confidence interval, $T R D$ treatment-resistant depression

${ }^{a}$ Includes safety evaluable patients from studies cited in references Daly et al. [4, 7], Fedgchin et al. [5], Popova et al. [6], and Ochs-Ross et al. [9]

${ }^{b}$ Includes safety evaluable patients from studies cited in Daly et al. [4, 7], Fedgchin et al. [5], Popova et al. [6], Wajs et al. [8], and Ochs-Ross et al. [9]

${ }^{c}$ Includes all patients who had $\geq 1$ occurrences of an adverse event that coded to the MedDRA ${ }^{\circledR}$ preferred terms grouped under 'Cardiovascular Adverse Events of Clinical Interest'; the patient is counted only once regardless of the number of events or the number of occurrences

${ }^{\mathrm{d}}$ The two-sided exact $95 \% \mathrm{CI}$ in odds ratio of esketamine + oral antidepressant to oral antidepressant + placebo for All Randomized, Blinded Trials TRD Population

${ }^{\mathrm{e}}$ Event was severe and serious, resulting in death

${ }^{\mathrm{f}}$ One event in the esketamine + oral antidepressant group and three events in the All Clinical Trials TRD Population were severe and non-serious ${ }^{g}$ One event was severe and serious, and one event was severe and non-serious

${ }^{\mathrm{h}}$ The total number of distinct preferred terms (i.e., preferred terms that refer to separate adverse events reported by individual patients) in the 'Cardiovascular Adverse Events of Clinical Interest' group by seriousness/outcome and also by severity

double-blind phases across the two short-term phase III studies in patients 18-64 years of age (pooled TRANSFORM-1 and -2 studies). Thirteen patients who were on esketamine in TRANSFORM-3 $(n=2)$, SUSTAIN-1 $(n=2)$, and SUSTAIN-2 $(n=9)$ discontinued study medication due to adverse events related to increased BP. Overall, discontinuation of esketamine treatment due to adverse events of increased BP and tachycardia occurred in $<2 \%$ of patients across studies/study phases.

In the phase II dose-ranging study, there was no evidence of a clear dose response across the three doses of esketamine in terms of BP changes. In the phase III fixed-dose study TRANSFORM-1, differences between the maximum mean changes in BP between the 56 and $84 \mathrm{mg}$ doses of 


\section{a Systolic Blood Pressure}

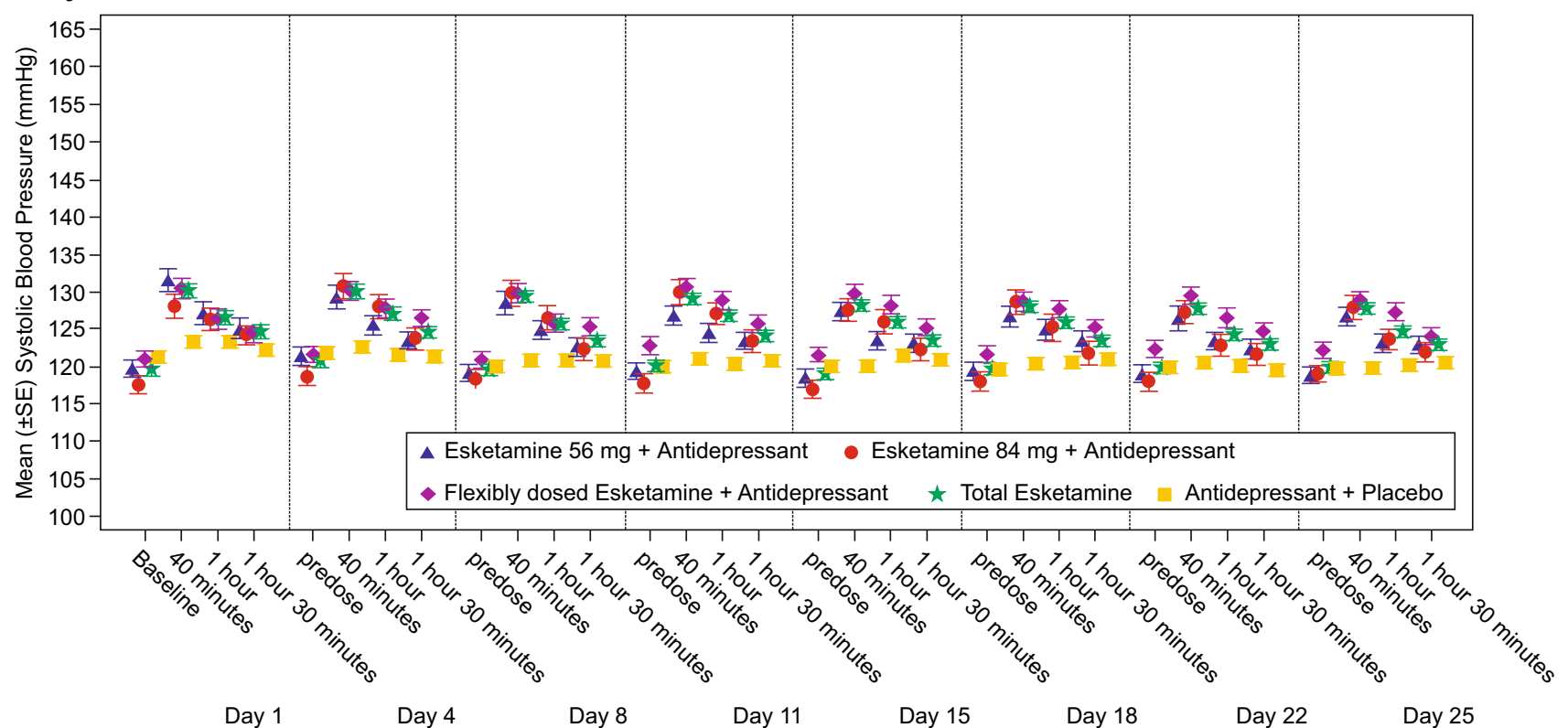

b Diastolic Blood Pressure

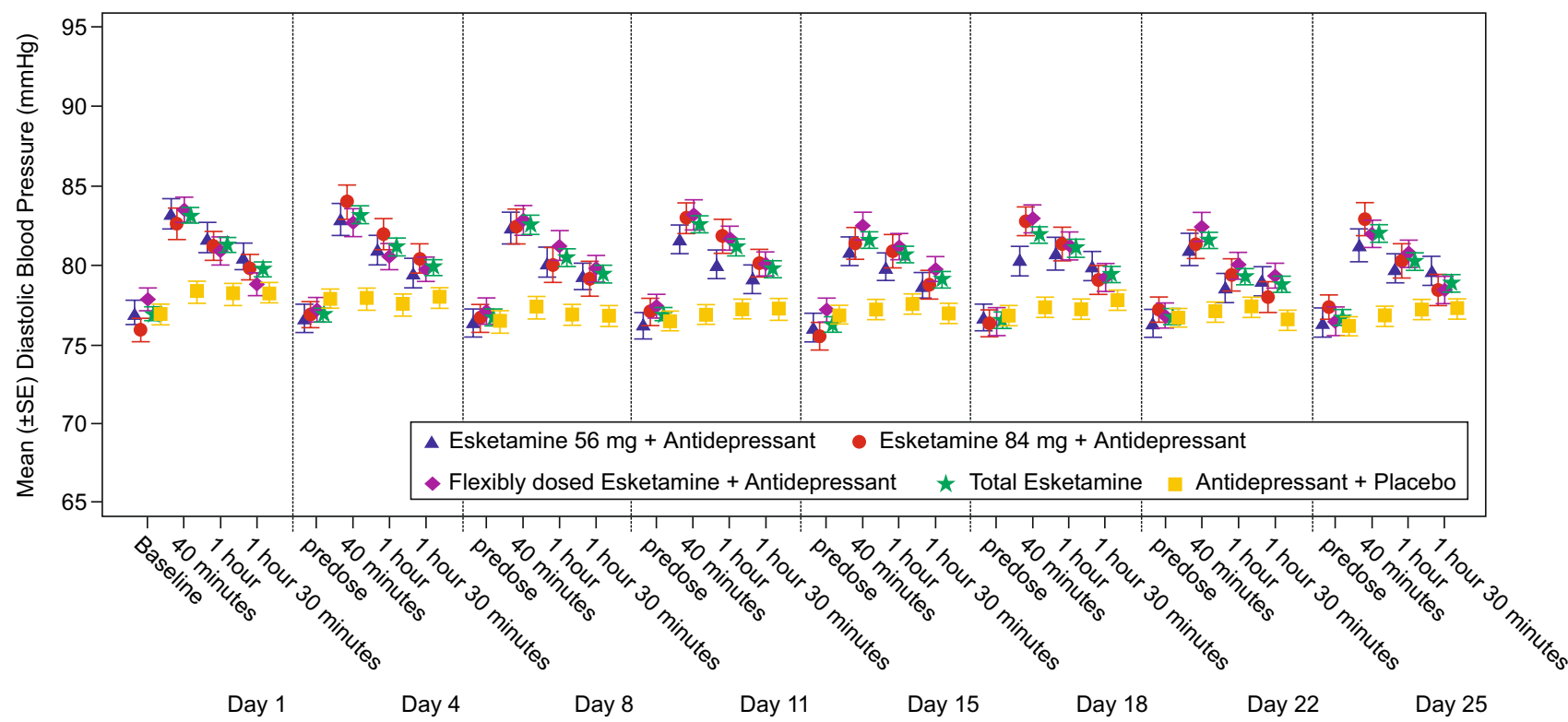

Fig. 1 Mean $[ \pm$ standard error (SE)] blood pressure over time from the induction phase of 4-week double-blind studies of patients with treatment-resistant depression (TRD) aged 18-64 years [5, 6]

esketamine were small and not suggestive of a dose response (14.3 and $15.0 \mathrm{mmHg}$, respectively, for SBP; 8.9 and $9.4 \mathrm{mmHg}$, respectively, for DBP).

The proportion of patients who initiated a new antihypertensive medication after an acute episode of hypertension was comparable between treatment groups in the short-term double-blind studies [one (of 17) esketamine/antidepressant patient vs. zero (of two) antidepressant/placebo patients]. Among the patients without a history of hypertension who participated in the short-term double-blind studies, new antihypertensive medication was initiated by $2.1 \%(6 / 280)$ of patients in the esketamine group [amlodipine: two $(0.7 \%)$, captopril: one $(0.4 \%)$, losartan: one $(0.4 \%)$, metoprolol: one $(0.4 \%)$, propranolol: one $(0.4 \%)$ versus $1.2 \%(2 / 171)$ of patients in the antidepressant/placebo group hydrochlorothiazide: one $(0.6 \%)$, prazosin: one $(0.6 \%)]$.

The proportion of postdose adverse events of increased BP that were treated with a rescue ('as needed') medication 
on the dosing day in the short-term double-blind studies was $2.2 \%$ (two of 93 events) in the esketamine/antidepressant group versus 0\% (zero of two events) in the antidepressant/ placebo group, and in the long-term open-label safety study, $2.4 \%$ (eight of 330 events).

An analysis of adverse events by class of antidepressant did not show any trend or clinically meaningful difference in cardiovascular or BP-related adverse events between patients taking SSRIs and those taking SNRIs. Of note, on dosing days the oral antidepressant was taken at least $3 \mathrm{~h}$ after the esketamine nasal spray session to alleviate the potential effect of oral antidepressants on postdose BP, especially for those oral antidepressants (e.g., venlafaxine) that are associ-

Across the six phase II/III clinical studies, one esketamine-treated patient, a 69 -year-old obese female with a medical history of hypertension, asthma, and diabetes mellitus, had a maximum postdose QTcF (QT corrected by Fridericia's equation) value exceeding $500 \mathrm{~ms}$ [predose QTcF of $406 \mathrm{~ms}$ and a postdose QTcF $(1 \mathrm{~h})$ of $527 \mathrm{~ms}$ ]. The increase in QTcF was reported as an adverse event leading to discontinuation. No adverse sequelae were reported. Ten other esketamine-treated patients had a maximum postdose QTcF value $>480-500 \mathrm{~ms}$; eight esketamine-treated patients had an increase of QTcF from a baseline value of $\geq 60 \mathrm{~ms}$. Clinically significant findings related to abnormal ECG parameters were reported as adverse events at low incidences for esketamine-treated patients and were generally similar among patients who received esketamine/antidepressant and those who received antidepressant/placebo in controlled studies. One patient, a 40-year-old male, in the long-term safety study (SUSTAIN-2) discontinued treatment due to an adverse event of ventricular arrhythmia, which manifested as premature ventricular complexes in the ECG.

\section{a Mean Maximum}
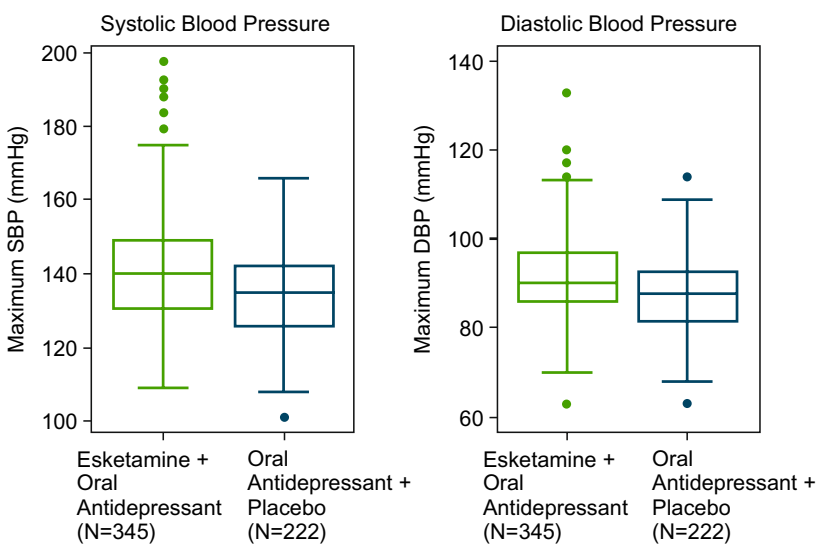
ated with increased BP.

In the phase I thorough QTc study, the mean difference (90\% CI) from placebo for change in baseline-corrected QTcF over the 30 -h postdose interval ranged from $-2.02 \mathrm{~ms}$ ( -4.173 to 0.137$)$ to $2.16 \mathrm{~ms}(0.018$ to 4.310$)$ for the $84 \mathrm{mg}$ intranasal esketamine dose and from $-3.51 \mathrm{~ms}(-5.721$ to -1.294 ) to $4.89 \mathrm{~ms}$ (2.676 to 7.102 ) for the $0.8 \mathrm{mg} / \mathrm{kg} \mathrm{IV}$ infusion (data on file). These results suggest that therapeutic and supratherapeutic doses of esketamine do not prolong the QTcF interval, which was below the $10 \mathrm{~ms}$ threshold specified in the International Conference on Harmonisation of Technical Requirements for Registration of Pharmaceuticals for Human Use (ICH) E14 guideline $[19,20]$ at all timepoints.

The evidence discussed here did not support esketamine having a proarrhythmic potential.

\section{Discussion}

The recent US FDA and EMA approvals of esketamine nasal spray as an antidepressant, in conjunction with an oral antidepressant, for patients with TRD has led to great interest in the field of psychiatry. Publications of phase II and III study data have focused on its efficacy and general safety [4-9]. With this report, the data suggest a low risk of serious cardiovascular outcomes when esketamine is administered intranasally in the clinic setting to patients with TRD and a history of no or controlled hypertension. BP elevations following dosing of esketamine nasal spray were generally transient, asymptomatic, self-limiting, without need for rescue medications, and not associated with serious cardiovascular safety sequalae. No clinically relevant effect on ECG parameters was observed in the esketamine development program.

In both double-blind treatment groups, elderly patients ( $\geq 65$ years) had larger increases in SBPs and DBPs than
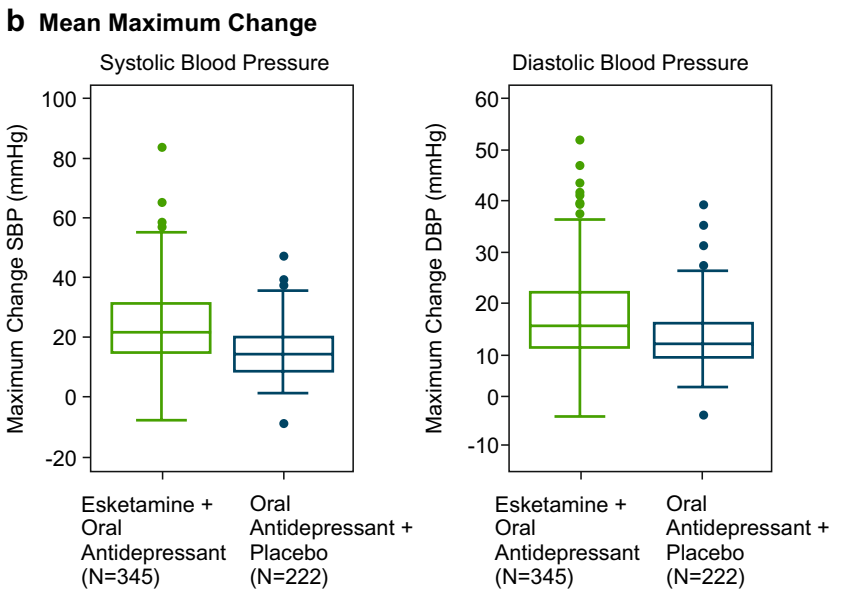

Fig. 2 Mean maximum [ \pm standard error (SE)] and maximum change in postdose blood pressure: pooled results from the induction phase of 4-week double-blind studies of patients with treatment-resistant depression (TRD) aged 18-64 years [5, 6] 
younger patients, more so among those treated with esketamine/antidepressant than with antidepressant/placebo. This finding is likely a consequence of increased arterial stiffness with advancing age [21]. This difference in cardiovascular response to esketamine did not appear to increase the risk of adverse cardiac events for elderly patients with TRD.

Almost half (47\%) of the 1708 patients exposed to esketamine in the study cohort were enrolled and assessed in a long-term safety study, with 479 patients ( $28 \%$ of the All Clinical Trials TRD Population) exposed to esketamine for at least 6 months. Another strength of our analysis is that the approach to assessment of cardiovascular safety was consistent across the phase II and III studies of esketamine, adding credence to combining patient-level data and confidence in the pooled summary statistics. That said, the generalizability of our study findings may be limited by the exclusion of participants with a history of cardiovascular disease within 12 months before the start of the trials, uncontrolled hypertension at study enrollment, or clinically significant ECG abnormalities. It should also be noted that although acute effects were monitored, potential BP elevations or ECG changes occurring between dosing sessions were not routinely documented. Further monitoring for long-term cardiovascular outcomes of this treatment is warranted. The long-term effects of repeated transient BP increases lasting $1-4 \mathrm{~h}$ are unknown.

The results described here provide reassurance on the cardiovascular safety of esketamine nasal spray, when administered to patients without hypertension or with wellcontrolled hypertension and under the observation of a healthcare provider, as directed by the Spravato ${ }^{\mathrm{TM}}$ prescribing information [18]. Patient screening and careful monitoring of $\mathrm{BP}$ and cardiovascular functioning are important during the time patients are receiving treatment with esketamine nasal spray. Patients with cardiovascular and cerebrovascular conditions should not be treated with esketamine if the risk outweighs the benefit in this population (details provided in the Spravato ${ }^{\mathrm{TM}}$ prescribing information [18]).

\section{Conclusions}

Our findings formed the basis for instructions in the Spravato $^{\mathrm{TM}}$ prescribing information [18] regarding predose $\mathrm{BP}$ thresholds, BP assessments (i.e., prior to and after dosing at approximately $40 \mathrm{~min}$ and subsequently, as clinically warranted), and recommended actions to manage BP elevation.

Acknowledgements We acknowledge Sandra Norris, PharmD of the Norris Communications Group LLC for medical writing assistance and Ellen Baum, PhD (Janssen Global Services, LLC) for additional editorial support. We also acknowledge Peter Zannikos, PhD, of Janssen Research \& Development, LLC for providing data from the phase I thorough QTc interval study of esketamine.
Author Contributions RM conducted the statistical analyses. All authors were involved in interpretation of the results and review of the manuscript. All authors meet ICMJE (International Committee of Medical Journal Editors) criteria and all those who fulfilled those criteria are listed as authors.

\section{Compliance with Ethical Standards}

Funding The open access fee was paid by Janssen Research \& Development, LLC, Titusville, NJ, USA, which also provided funding for the studies reported in this article.

Conflict of interest Teodora Doherty, Ewa Wajs, Rama Melkote, and Janice Miller are employees of Janssen Research \& Development, LLC and hold company equity. Michael A. Weber is a consultant with Janssen Pharmaceuticals. Jaskaran B. Singh worked on the clinical development program of esketamine for treatment-resistant depression during his employment by Janssen Research \& Development, LLC; he is currently employed by Neurocrine Biosciences, San Diego, CA USA.

Consent for publication All authors provided their consent for publication of this article.

Availability of data The data sharing policy of Janssen Pharmaceutical Companies of Johnson \& Johnson is available at https://www.janss en.com/clinical-trials/transparency. As noted on this site, requests for access to the study data can be submitted through Yale Open Data Access (YODA) Project site at http://yoda.yale.edu.

Ethics approval and consent to participate An Institutional Review Board (IRB) (USA) or Independent Ethics Committee (IEC) (Europe) approved the study protocols and their amendments. All procedures performed in the studies were in accordance with the ethical standards of the IRB/IEC and/or national research committee and with the 1964 Helsinki Declaration and its later amendments or comparable ethical standards. Informed consent was obtained from all individual participants included in the studies.

Open Access This article is licensed under a Creative Commons Attribution-NonCommercial 4.0 International License, which permits any non-commercial use, sharing, adaptation, distribution and reproduction in any medium or format, as long as you give appropriate credit to the original author(s) and the source, provide a link to the Creative Commons licence, and indicate if changes were made. The images or other third party material in this article are included in the article's Creative Commons licence, unless indicated otherwise in a credit line to the material. If material is not included in the article's Creative Commons licence and your intended use is not permitted by statutory regulation or exceeds the permitted use, you will need to obtain permission directly from the copyright holder. To view a copy of this licence, visit http://creativecommons.org/licenses/by-nc/4.0/.

\section{References}

1. Newport DJ, Carpenter LL, McDonald WM, Potash JB, Tohen M, Nemeroff CB, APA Council of Research Task Force on Novel Biomarkers and Treatments. Ketamine and other NMDA antagonists: early clinical trials and possible mechanisms in depression. Am J Psychiatry. 2015;172(10):950-66.

2. Zarate CA Jr, Singh JB, Carlson PJ, Brutsche NE, Ameli R, Luckenbaugh DA, et al. A randomized trial of an $N$-methyl-D-aspartate 
antagonist in treatment-resistant major depression. Arch Gen Psychiatry. 2006;63(8):856-64.

3. Murrough JW, Iosifescu DV, Chang LC, Al Jurdi RK, Green CE, Perez AM, et al. Antidepressant efficacy of ketamine in treatmentresistant major depression: a two-site randomized controlled trial. Am J Psychiatry. 2013;170(10):1134-42.

4. Daly EJ, Singh JB, Fedgchin M, Cooper K, Lim P, Shelton RC, et al. Efficacy and safety of intranasal esketamine adjunctive to oral antidepressant therapy in treatment-resistant depression: a randomized clinical trial. JAMA Psychiatry. 2018;75(2):139-48.

5. Fedgchin M, Trivedi MH, Daly EJ, Melkote R, Lane R, Lim P, et al. Efficacy and safety of fixed-dose esketamine nasal spray combined with a new oral antidepressant in treatment-resistant depression: results of a randomized, double-blind, active-controlled study (TRANSFORM-1). Int J Neuropsychopharmacol. 2019;22(10):616-30.

6. Popova V, Daly EJ, Trivedi M, Cooper K, Lane R, Lim P, et al. Efficacy and safety of flexibly-dosed esketamine nasal spray combined with a new oral antidepressant in treatment-resistant depression: results of a randomized, double-blind, active-controlled study. Lancet Psychiatry. 2019;176(6):428-38.

7. Daly EJ, Trivedi MH, Janik A, Li H, Zhang Y, Li X, et al. A double-blind, randomized withdrawal, multicenter study of esketamine nasal spray plus an oral antidepressant for relapse prevention in treatment-resistant depression (SUSTAIN-1). JAMA Psychiatry. 2019;76(9):893-903. https://doi.org/10.1001/jamapsychi atry.2019.1189.

8. Wajs E, Aluisio L, Holder R, Daly E, Lane R, Lim P, et al. Esketamine nasal spray plus oral antidepressant in patients with treatment-resistant depression: Assessment of long-term safety in a phase 3, open-label study (SUSTAIN-2). J Clin Psychiatry. 2020 (in press).

9. Ochs-Ross R, Daly EJ, Zhang Y, Lane R, Lim P, Hough D, et al. Efficacy and safety of esketamine nasal spray plus an oral antidepressant in elderly patients with treatment-resistant depressionTRANSFORM-3. Am J Geriatric Psychiatry. 2019. https://doi. org/10.1016/j.jagp.2019.10.008.

10. Traber DL, Wilson RD, Priano LL. Differentiation of the cardiovascular effects of CI-581. Anesth Analg. 1968;47(6):769-78.
11. Miller R, Eriksson L, Fleisher L, Wiener-Kronish J, Cohen N, Young W. Miller's anesthesia. 8th ed. Philadelphia: Elsevier; 2015.

12. Baum VC, Tecson ME. Ketamine inhibits transsarcolemmal calcium entry in guinea pig myocardium: direct evidence by single cell voltage clamp. Anesth Analg. 1991;73(6):804-7.

13. Riva-Posse P, Reiff CM, Edwards JA, Job GP, Galendez GC, Garlow SJ, et al. Blood pressure safety of subanesthetic ketamine for depression: a report on 684 infusions. J Affect Disord. 2018;236:291-7.

14. Wan LB, Levitch CF, Perez AM, Brallier JW, Iosifescu DV, Chang LC, et al. Ketamine safety and tolerability in clinical trials for treatment-resistant depression. J Clin Psychiatry. 2015;76(3):247-52.

15. Mrazek DA, Hornberger JC, Altar CA, Degtiar I. A review of the clinical, economic, and societal burden of treatment-resistant depression: 1996-2013. Psychiatr Serv. 2014;65(8):977-87.

16. van Melle JP, de Jonge P, Spijkerman TA, Tijssen JG, Ormel J, van Veldhuisen DJ, et al. Prognostic association of depression following myocardial infarction with mortality and cardiovascular events: a meta-analysis. Psychosom Med. 2004;66(6):814-82.

17. Carney RM, Freeland KE. Depression, mortality, and medical morbidity in patients with coronary heart disease. Biol Psychiatry. 2003;54(3):241-7.

18. Spravato ${ }^{\mathrm{TM}}$ (esketamine) nasal spray prescribing information. Titusville: Janssen Pharmaceutical Companies; 2019.

19. Food and Drug Administration. Guidance for Industry: E14 clinical evaluation of QT/QTc Interval prolongation and proarrhythmic potential for non-antiarrhythmic drugs; October 2005. https ://www.fda.gov/downloads/Drugs/GuidanceComplianceRegulat oryInformation/Guidances/ucm073153.pdf. Accessed 30 Oct 2019.

20. Food and Drug Administration. Guidance for Industry. E14 Clinical evaluation of QT/QTc interval prolongation and proarrhythmic potential for non-antiarrhythmic drugs-questions and answers (R3); June 2017. https://www.fda.gov/downloads/Drugs/Guida nceComplianceRegulatoryInformation/Guidances/UCM073161. pdf.

21. Van Bortel LM, Spek JJ. Influence of aging on arterial compliance. J Hum Hypertens. 1988;12(9):583-6.

\title{
Affiliations
}

\author{
Teodora Doherty ${ }^{1} \cdot$ Ewa Wajs $^{2} \cdot$ Rama Melkote $^{3} \cdot$ Janice Miller $^{1} \cdot$ Jaskaran B. Singh ${ }^{4,6} \cdot$ Michael A. Weber $^{5}$ \\ 1 Neuroscience, Global Medical Organization, Janssen \\ Research \& Development, LLC, Titusville, NJ, USA \\ 2 Janssen Research \& Development, Belgium, Beerse, Belgium \\ 3 Janssen Research \& Development, LLC, Raritan, NJ, USA \\ 4 Janssen Research \& Development, LLC, San Diego, CA, \\ USA \\ 5 State University of New York Downstate College \\ of Medicine, Brooklyn, NY, USA \\ 6 Neurocrine Biosciences, San Diego, CA, USA
}

